\title{
The immunoregulation of mesenchymal stem cells plays a critical role in improving the prognosis of liver transplantation
}

Chenxia $\mathrm{Hu}^{1,2}$ and Lanjuan $\mathrm{Li}^{1,2^{*}}$

\begin{abstract}
The liver is supplied by a dual blood supply, including the portal venous system and the hepatic arterial system; thus, the liver organ is exposed to multiple gut microbial products, metabolic products, and toxins; is sensitive to extraneous pathogens; and can develop liver failure, liver cirrhosis and hepatocellular carcinoma (HCC) after short-term or long-term injury. Although liver transplantation (LT) serves as the only effective treatment for patients with end-stage liver diseases, it is not very popular because of the complications and low survival rates. Although the liver is generally termed an immune and tolerogenic organ with adaptive systems consisting of humoral immunity and cell-mediated immunity, a high rejection rate is still the main complication in patients with LT. Growing evidence has shown that mesenchymal stromal cell (MSC) transplantation could serve as an effective immunomodulatory strategy to induce tolerance in various immune-related disorders. MSCs are reported to inhibit the immune response from innate immune cells, including macrophages, dendritic cells (DCs), natural killer cells (NK cells), and natural killer T (NKT) cells, and that from adaptive immune cells, including $T$ cells, B cells and other liver-specific immune cells, for the generation of a tolerogenic microenvironment. In this review, we summarized the relationship between LT and immunoregulation, and we focused on how to improve the effects of MSC transplantation to improve the prognosis of LT. Only after exhaustive clarification of the potential immunoregulatory mechanisms of MSCs in vitro and in vivo can we implement MSC protocols in routine clinical practice to improve LT outcome.
\end{abstract}

Keywords: Mesenchymal stromal cell, Immunoregulation, Liver transplantation, Rejection, Prognosis

\section{Background}

The liver is supplied by a dual blood supply, including the portal venous system and the hepatic arterial system; thus, the liver organ is exposed to multiple gut microbial products, metabolic products, and toxins; is sensitive to extraneous pathogens; and can develop liver failure, liver cirrhosis and hepatocellular carcinoma (HCC) after short-term or long-term injury. Early in 1963, the first case of liver transplantation (LT) was performed by Dr.

\footnotetext{
*Correspondence: ljli@zju.edu.cn

${ }^{1}$ Collaborative Innovation Center for Diagnosis and Treatment

of Infectious Diseases, State Key Laboratory for Diagnosis and Treatment of Infectious Diseases, First Affiliated Hospital, School of Medicine,

Zhejiang University, Hangzhou, Zhejiang, People's Republic of China

Full list of author information is available at the end of the article
}

Thomas Starzl for irreversible injury, but it was not very popular because of the complications and low survival rates throughout the 1960s and 1970s [1]. Although the liver is generally termed an immune and tolerogenic organ with adaptive systems consisting of humoral immunity and cell-mediated immunity, a high rejection rate is still the main complication in patients with LT [2]. Moreover, acute graft-versus-host disease, which is induced by the interaction of the innate and adaptive immune systems, is a serious and life-threatening complication of LT that occurs in $1 \%$ to $2 \%$ of liver allograft recipients. Thus, therapies targeting immune cells may be beneficial for transplanted grafts and protect against severe rejection processes. Although other factors, such as secondary infection and unstable surgical techniques,

(c) The Author(s) 2019. This article is licensed under a Creative Commons Attribution 4.0 International License, which permits use, sharing, adaptation, distribution and reproduction in any medium or format, as long as you give appropriate credit to the original author(s) and the source, provide a link to the Creative Commons licence, and indicate if changes were made. The images or other third party material in this article are included in the article's Creative Commons licence, unless indicated otherwise in a credit line to the material. If material is not included in the article's Creative Commons licence and your intended use is not permitted by statutory regulation or exceeds the permitted use, you will need to obtain permission directly from the copyright holder. To view a copy of this licence, visit http://creativecommons.org/licenses/by/4.0/. The Creative Commons Public Domain Dedication waiver (http://creativecommons.org/publicdomain/zero/1.0/) applies to the data made available in this article, unless otherwise stated in a credit line to the data.. 
also influence liver graft and patient survival, the main issue is the determination of safe and effective immunosuppression agents. Cyclosporine emerged as an effective immunosuppressant that obviously reduced the rejection rate and prolonged the survival time of LT recipients [3]. However, the application of immunosuppressive agents contributes to metabolic complications, inevitable viral recurrence, and opportunistic infections in LT recipients [4].

Growing evidence has shown that mesenchymal stromal cell (MSC) transplantation could serve as an effective immunomodulatory strategy to induce tolerance in various immune-related disorders. The ISCT committee set a definition of MSCs as follows: MSCs are plasticadherent and fibroblast-like after culture in vitro; they are positive for surface molecules such as CD105, CD73 and CD90 but negative for surface molecules such as CD45, CD34, CD14 (or CD11b), CD79alpha (or CD19) or human leukocyte antigen (HLA)-DR by flow cytometry; and they can be differentiated into adipocytes, osteocytes and chondrocytes in vitro [5]. These multipotent cells are generally isolated from various tissues, including bone marrow, adipose, umbilical cord, tooth pulp, and cord and participate in the regulation of organ homeostasis, tissue remodeling and damage repair [6]. They are immune-privileged in vivo since they have low expression of class II major histocompatibility complex (MHC)II and costimulatory molecules [7]. MSCs are able to migrate into injured liver sites, undergo proliferation and hepatic differentiation, secrete anti-inflammatory factors and interact with immune cells to repair liver injury and prohibit liver failure [8]. Intriguingly, MSCs participate in generating a balanced microenvironment via cell-cell interactions and paracrine pathways. Thus, MSC transplantation serves as a novel treatment regimen for preventing graft rejection and treating autoimmune diseases such as graft-versus-host disease via their immunomodulatory effects [9].

In this review, we summarized the relationship between LT and immunoregulation, and we focused on how to improve the effects of MSC transplantation to improve the prognosis of LT. Then, we highlight that the time points of MSC administration or preconditioning with anti-inflammatory factors or gene modification further improve the effects of MSC-based therapies in LT grafts or recipients. Only after exhaustive clarification of the potential mechanisms of MSCs on immunoregulation in vitro and in vivo can we implement MSC protocols in routine clinical practice to improve LT outcome.

\section{Activation of innate or adaptive immune cells in vivo}

Both innate immune cells and adaptive immune cells generated from bone marrow-derived progenitor cells constitute the immune system in mammals. In response to external pathogens, innate immune cells respond more rapidly than adaptive immune cells. Pattern recognition receptors and cytokine receptors quickly activate innate immune cells to lyse foreign pathogens and generate various cytokines for further activation of adaptive immune cells.

\section{Activation of innate immune cells in vivo}

The innate immune system consists of macrophages, dendritic cells (DCs), natural killer cells (NK cells), and natural killer $\mathrm{T}$ (NKT) cells. All these immune cells are able to activate the adaptive immune cells through cell-cell interaction and secretion of chemokines and cytokines [10]. Macrophages are immune cells that can be generated from tissue resident macrophages and circulating macrophages from bone marrow [11]. Macrophages can be polarized into proinflammatory M1 or anti-inflammatory M2 macrophages. M1 macrophages produce multiple inflammatory factors, including tumor necrosis factor (TNF)- $\alpha$, interleukin (IL)-1 $\beta$, CCL2, and CXCL1, to generate and promote tissue damage after they are activated by toll-like receptor (TLR) agonists, pathogen-associated molecular patterns (PAMPs) and damage-associated molecular patterns (DAMPs) in response to changed microenvironments [12]. M1 macrophages will switch to IL-10-secreting M2 macrophages to decrease tissue injury and resolve inflammation in response to dead cells and several inflammatory factors, including IL-4 and IL-13 [13]. DCs are important innate immune cells that serve as antigen-presenting cells (APCs) to present and process external antigens to secondary lymphoid tissues, such as the spleen and lymph nodes, for further activation of adaptive immune cells. The DCs are divided into conventional DCs and plasmacytoid DCs under steady state in vivo, while they change their phenotype and functionality according to different inflammatory conditions. Although the immune function of plasmacytoid DCs is weaker than that of mature DCs, plasmacytoid DCs are a subset of DCs that can produce IL-10 and TGF- $\beta$ to induce the differentiation of T cells into Tregs via the TLR7/9 pathway [14]. Activated DCs selectively secrete various cytokines, including IL-12, IL-23 and Notch ligands, in response to the inflammatory microenvironment to induce the differentiation of activated $\mathrm{T}$ cells into $\mathrm{T}$ helper 1 (Th1), Th2 and Th17 cells [15]. In response to inflammatory stimulation, the quiescent and tolerant DCs will transform into inflammatory DCs and induce T cell-mediated immune responses [16]. 
Intake of apoptotic DCs enabled the conversion of immature DCs into tolerogenic DCs to promote the differentiation of $\mathrm{T}$ cells into forkhead box P3 (Foxp3)+ Tregs [17]. NK cells are innate immune cells that directly kill injured cells by natural cytotoxicity and secretion of interferongamma (IFN- $\gamma$ ). NK cells are able to distinguish self- and non-self for innate and adaptive immune responses and participate in the lysis of harmful pathogens in allografts [18]. Intriguingly, NKT cells are a type of noncirculating, tissue-resident lymphocyte with innate immunity and adaptive immunity. These specific immune cells express both the T cell receptor (TCR) $\alpha$-chain and the surface receptors of NK cells for the regulation of immunoregulation [19]. NKT cells recognize lipids present on CD1d and secrete various cytokines after differentiation into distinct subsets that resemble subsets of CD4+ T helper cells including Th1, Th2 and Th17 cell subsets and subsets of innate lymphocyte cells (ILCs). Activated NKT1 cells are similar to Th1 cells and group 1 ILCs (ILC1s), which express high levels of T-bet, IL-4 and IFN- $\gamma$. NKT2 cells are similar to Th2 cells, which secrete high levels of cytokines, including IL-4, IL-5 and IL-13, and NKT17 cells resemble Th17 cells, which secrete high levels of cytokines including IL-17, IL-22, granulocyte macrophage colony stimulating factor (GM-CSF) and TNF [20].

\section{Activation of adaptive immune cells in vivo}

In comparison to the innate immune system, the activation of $T$ and $B$ cells leads to selective expression of several types of antigen receptors that shape their specific functions in immunoregulatory processes. Moreover, TCR specifically binds antigens in the context of MHC molecules, while B cell antigen receptor (BCR) binds antigens in an MHC-independent manner. The costimulatory receptor engagement maximally activates the innate and adaptive immune systems to clear pathogens and respond rapidly to reinfection [21]. In addition, TCRs and other costimulatory factors are responsible for the activation of naïve $\mathrm{T}$ cells in vivo, thus protecting against infection-induced cytotoxicity and immunemediated diseases. Different stimulations will promote the differentiation of activated $\mathrm{CD} 4+\mathrm{T}$ cells into $\mathrm{T}$ helper cells and regulatory $\mathrm{T}$ cells (Tregs) [22]. On the other hand, multiple extraneous pathogens transform CD8+ T cells into cytotoxic $\mathrm{T}$ lymphocytes, which promote the lysis of injured cells via secretion of granzymes, perforins, and cytokines [23]. Albano et al. [24] demonstrated that $\mathrm{CD} 4+\mathrm{T}$ helper cells were indispensable for activation of cytotoxic CD8+ T cells and B lymphocytes and differentiation of macrophages to eliminate cells infected with external pathogens. B cells, a subset of lymphocytes from bone marrow, take part in the regulation of T-cell-dependent and T-cell-independent immunoregulation. B cells effectively recognized exotic antigens and then proliferated and differentiated into antibody-producing $B$ lymphocytes and memory $B$ lymphocytes to protect against pathogen-induced injury in vivo [25]. B lymphocytes are divided into B1 cells, which are enriched in the pleural and peritoneal cavities, and B2 cells, which are conventional B lymphocytes. Moreover, regulatory B cells (Bregs) are another subset of B lymphocytes that can produce IL-10 and participate in the induction of tolerance in vitro and in vivo [26].

\section{Regulation of immune cells in response to LT}

The liver is an immunotolerant tissue with various cells expressing low levels of MHC antigens, and it is difficult to induce an innate or adaptive immune response in the liver $[27,28]$. Multiple immune cells, such as T cells, B cells, NK cells, NKT cells, liver sinusoidal endothelial cells (LSECs), Kupffer cells (KCs) and DCs, are located in liver tissue and migrate from peripheral blood for recognition and response to pathogens in an antigen-specific manner. Intrahepatic immune cells exert high immunosuppressive effects via cell-cell interactions and secretion of immunosuppressive cytokines after LT.

Two sources of immune cells, donor liver-resident cells and recipient immune cells, respond to the altered microenvironment after LT. The former immune cells are those graft-derived immune cells that enter the peripheral blood of recipients, and the latter are those recipientderived immune cells that invade into liver grafts [29]. $\mathrm{T}$ cells and $\mathrm{B}$ cells first recognize pathogens and then take part in antigen-presenting activities. Allospecific $\mathrm{T}$ cells recognize foreign MHC molecules on donor tissue cells and play a critical role in the rejection of solid organ grafts [30]. Kim et al. [31] showed that calcineurin inhibitor-based immunosuppression maintained effector $T$ or memory $B$ cells during the early posttransplantation period accompanied by suppression of Tregs.

In normal adult liver tissue, NK cells are the most abundant immune cells in all liver-derived lymphocytes. NK cells may participate in the induction of immune tolerance, as García et al. [32] showed that recipients with immune tolerance have higher levels of NK cells than those with immune rejection in their peripheral blood. The liver graft-derived recipient NK cells generate tolerant phenotypes after decreasing receptors, cytotoxicity and cytokine secretion via perturbation of the IL-12/ STAT-4 signaling pathway after LT [33]. However, there is debate about the effects of NK cells on LT recipients. Depletion of NK cells or inhibition of IFN significantly upregulated the survival rate of liver grafts; thus, therapy targeting NK cells and their secretion of cytokines may contribute to the improvement in LT prognosis [34]. 
LSECs that constitute the wall of the hepatic sinusoid are the main nonparenchymal cells in liver tissue, while there are only a few reticular fibers attached to them that express only a few of the MHC-II molecules. LSECs switch into proinflammatory and prothrombotic states and vasoconstrict after the excised liver grafts were preserved by cold storage prior to LT. The unabridged functions are critical to the outcome of LT according to the current study [35]. Intriguingly, these nonparenchymal cells interact with other immune cells for antigen presentation and elimination of harmful immune responses in vivo. The antigen presentation of LSECs induced immunological tolerance rather than enhancement of immunoregulation against cytotoxic antigens through $\mathrm{CD} 8+\mathrm{T}$ cells, thus leading to suppression of the immunological response in liver [36]. LESCs significantly inhibited the proliferation of CD4+ T cells and IL- 2 generation, and they further induced the apoptosis of CD4+ $\mathrm{T}$ cells via the Fas-FasL pathway [37]. In addition, LSECs further promoted the differentiation of CD4+ T cells into CD25lowFoxp3- Tregs, which decreased the number of infiltrated inflammatory cells in liver tissue [38]. Twenty percent of the nonparenchymal cells in the liver tissue are residential macrophages (KCs), and these cells are located in the liver sinusoid and participate in the phagocytosis process via secretion of cytokines including IL-1, IL-6, IL-12, IL-18, TNF- $\alpha$, and IFN- $\gamma$ and presentation of antigens $[39,40]$. Liver KCs were reported to activate the TLR2/4 pathway and secrete IL-10 for suppression of IL-18-dependent NK cell activation [41]. Liver graft rejection was related to high levels of IL-2, IFN- $\gamma$ and TNF- $\alpha$ and low levels of IL-10, while immune tolerance was induced by KCs with high PD-L1 levels; these KCs can directly contact $\mathrm{T}$ cells and decrease the proliferation and functions of $\mathrm{T}$ cells after LT to reduce acute rejection [42]. KCs are able to promote the apoptosis of $\mathrm{T}$ cells through the Fas/FasL pathway [39], and Chen et al. [43] showed that pretreatment with KCs in the recipients before LT significantly decreased the number of apoptotic hepatocytes and attenuated the liver injury content in recipients with LT. In addition, KCs also promote Treg proliferation and secretion of IL-10 directly to inhibit the immune response of cytotoxic $\mathrm{T}$ lymphocytes on antigens targeting liver tissue [44]. Liver-derived DCs significantly inhibited the $\mathrm{T}$ cell response and promoted Treg activation to generate a definite immune tolerance via secretion of IL-10 [45]. The infusion of Tregs and immature DCs decreased the levels of total bilirubin and alanine aminotransferase and prolonged liver allograft survival of heterotopic LT rats via inducing alloantigen tolerance, as shown by upregulation of IL-10 and transforming growth factor (TGF)- $\beta 1$ and downregulation of IL-12 [46].
The balance between inflammation and anti-inflammation in liver tissue is responsible for the short- and long-term outcomes of LT. Modification of immune cells by immunosuppressive drugs, cytokines, stem cells or other pathways in vivo may decrease the rejection rate of liver grafts in recipients with LT. Thus, moderate immunosuppression by MSC transplantation with minimal adverse effects may prevent rejection and graft loss in LT recipients.

\section{The interaction between MSCs and immune cells}

Mesenchymal stromal cells can establish a stable and balanced microenvironment via regulation of innate or adaptive immune cells. The cell-cell interaction and specific secretome between MSCs and immune cells can guarantee the successful treatment of immune-related diseases. MSCs interact with innate and adaptive immune cells to regulate inflammation in vivo and in vitro. MSCs are reported to inhibit the immune response from macrophages, DCs, NK cells, NKT cells, T cells, Tregs, B cells and Bregs to generate a tolerogenic microenvironment (Fig. 1).

\section{The interaction between MSCs and innate immune cells}

Mesenchymal stromal cells effectively decrease IL-2-induced proliferation, cytotoxicity, and cytokine secretion (IFN- $\gamma$, IL-10 and TNF- $\alpha$ ) in activated NK cells in an IDO- and PGE2-dependent manner [47-49]. However, Casado et al. [50] highlighted that MSCs can only impair NK cell cytotoxicity via cell-cell contact. On the other hand, MSCs significantly prohibited the expansion, proliferation and IFN- $\gamma$ secretion in invariant NKT cells [51]. Liu et al. [52] demonstrated that MSCs significantly upregulated the recruitment of macrophages into injured sites for treating immune disorders and enhancing the repair of tissue injury. MSCs with high IDO activity effectively promoted the generation of anti-inflammatory M2 macrophages to block $\mathrm{T}$ cell activation; thus, $\mathrm{T}$ cell inhibition further amplified the immunosuppressive effects of MSCs [53]. It has been reported that MSCs also inhibited the generation and antigen presentation of peripheral blood monocyte-derived DCs after inhibiting cytokine release, differentiation and maturation of DCs $[54,55]$. MSCs and MSC-derived supernatants inhibited the activation and maturation of DCs by downregulating endocytosis and IL-12 production in DCs and then inhibited the activation of alloreactive $\mathrm{T}$ cells in vitro [54]. Furthermore, MSCs are able to inhibit the immunogenicity and $\mathrm{T}$ cell activation capacity of regulatory DCs, and these DCs can subsequently activate Tregs and secrete the anti-inflammatory factor IL-10 [56]. 


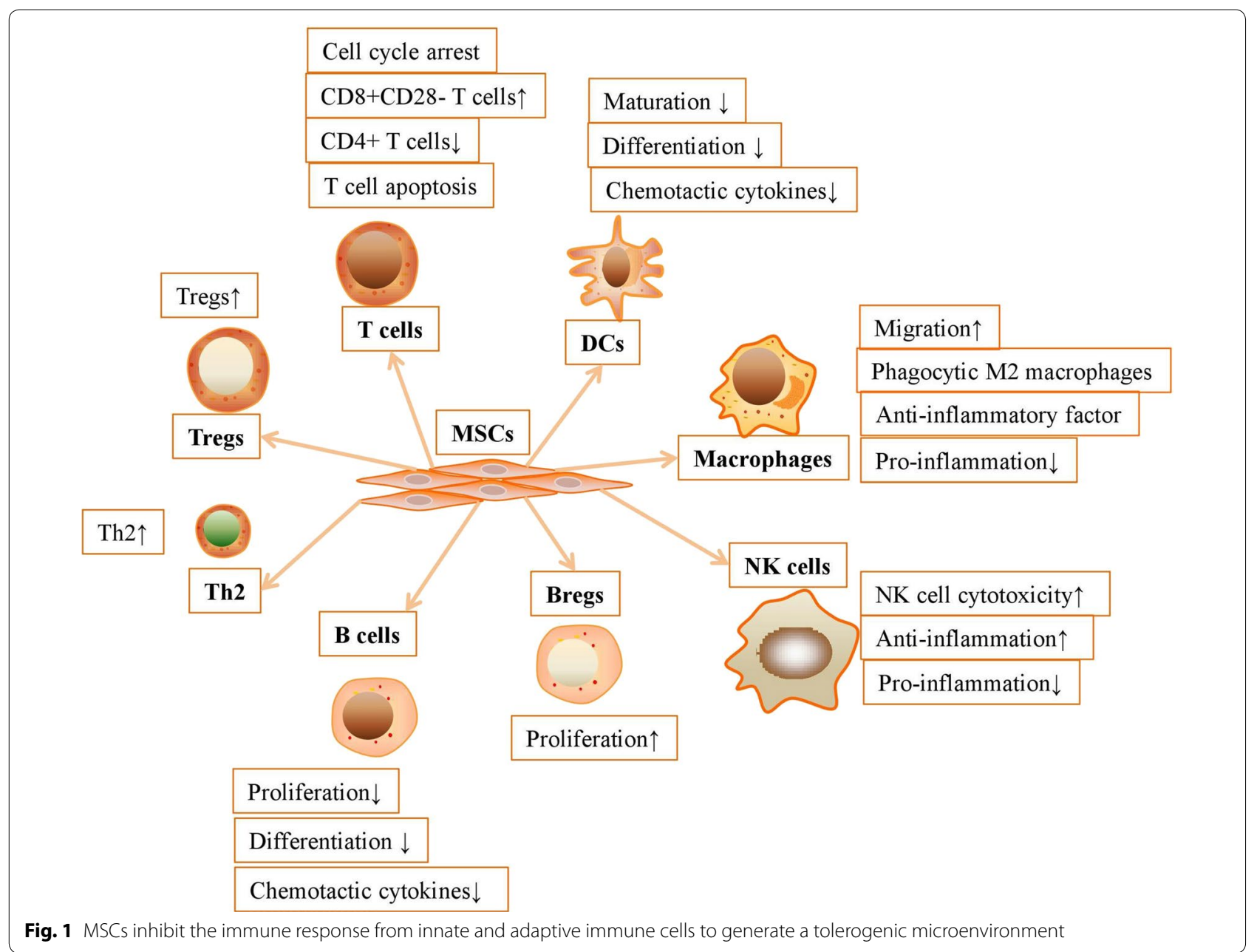

The interaction between MSCs and adaptive immune cells In addition to the innate immune system, MSCs significantly induced cell cycle arrest in $\mathrm{T}$ cells after downregulation of cyclin D2 and upregulation of p27kip1 in vitro [57]. MSCs upregulated the number of CD8+CD28- $\mathrm{T}$ cells, subsequently inhibiting the proliferation and activation of CD4 $+\mathrm{T}$ cells via downregulation of IFN- $\gamma$ and enhancing the apoptosis of activated CD4 $+\mathrm{T}$ cells [58]. The apoptosis of $\mathrm{T}$ cells is closely related to the conversion of tryptophan into kynurenine via an IDO-dependent pathway in the presence of IFN- $\gamma$ [59]. However, several investigators have highlighted that MSCs are only able to suppress $\mathrm{T}$ cell activity after pretreatment with inflammatory factors, such as IFN- $\gamma$, TNF- $\alpha$, IL- $1 \alpha$ or IL- $1 \beta$ [60]. These preconditioned MSCs participated in immunoregulation by significantly upregulating the expression of inducible nitric oxide synthase (iNOS) and cyclooxygenase 2 for the generation of nitric oxide (NO) and prostaglandin E2 (PGE2) [61]. In addition, MSCs secreted multiple cytokines and chemokines for recruitment of $\mathrm{T}$ cells into injured sites for immunosuppression and injury repair [62]. Furthermore, MSCs are reported to exert their immunoregulatory capacity by Treg induction indirectly, as Prevosto et al. [63] showed that MSCs significantly inhibited $\mathrm{T}$ lymphocytes after promoting the generation of Tregs from CD4+ or CD8+ T lymphocytes. However, Jiang et al. [64] debated that depletion of CD4+ CD25+ Tregs did not affect the immunosuppression of MSCs on CD4+ T cells, and they concluded that MSCs were not as important in Treg regulation. In a coculture system, MSCs transformed Th1 cells with high levels of proinflammatory factors (IL-2 and IFN- $\gamma$ ) into Th2 cells with high levels of anti-inflammatory factors (IL-4 and IL-10) [65].

Mesenchymal stromal cells were reported to inhibit the B lymphocyte-related humoral immune response via blocking the proliferation, differentiation and chemotactic cytokine production of B cells [66]. Peng et al. [67] demonstrated that infusion of MSCs significantly 
increased the survival time of patients with refractory chronic graft-versus-host disease through IDO-induced IL-10 secretion and Breg proliferation. Moreover, MSCs induced the generation of phagocytic M2 macrophages, which are able to secrete a large amount of anti-inflammatory factor IL-10, accompanied by low levels of inflammatory factors (IL-6, TNF- $\alpha$ and IL-1 $\beta$ ) $[68,69]$.

\section{MSC transplantation for improvement in LT prognosis}

Acute rejection is commonly encountered in LT recipients and may impact their long-term survival if rejection is severe or recurrent. Acute rejection after LT is usually treated with large doses of immunosuppressants with severe and toxic side effects, so it is imperative to find a safe and effective method for preventing rejection in LT recipients. Although MSCs significantly suppress the immune response by cell-cell interactions and the secretion of various cytokines after allogenic LT, pretreatments with growth factors or gene modification on MSCs can more obviously improve the prognosis of LT via regulation of the immune system (Table 1).

\section{The potential mechanisms of MSCs in improving the survival rate of $\mathrm{LT}$}

Currently, MSCs exert their immunoregulatory capacities to suppress the immune response by cell-cell interactions and the secretion of various cytokines after allogenic LT (Fig. 2).

Mesenchymal stromal cells significantly upregulated the survival rate of animals with LT via suppression of $\mathrm{KC}$ apoptosis, hepatocyte apoptosis, Th1/Th17 immune responses, chemokine expression and inflammatory cell infiltration [70]. MSCs are reported to inhibit the proliferation of CD4+ $\mathrm{T}$ cells and activation of CD8+ $\mathrm{T}$ cells in LT recipients on postoperative day 7 [71]. MSC transplantation markedly decreased the alanine aminotransferase level and improved allograft histology via activation of Tregs and Th2 cells and inhibition of Th1 and Th17 cells in animals with LT [72, 73]. Moreover, MSC administration inhibited allograft rejection and prolonged the survival time of $\mathrm{LT}$ rats via activation and expansion of CD4+CD25+Foxp3+ Tregs [74].

In addition to the immunoregulation of liver immune cells in vivo, MSC transplantation protects recipients of LT from acute rejection-induced injury via paracrine mechanisms. MSC administration upregulated the levels of TGF- $\beta 1$, Foxp3, IL-10, and CTLA- 4 on postoperative day 7 in rats with LT, while it downregulated the levels of TGF- $\beta 1$ and Foxp 3 on postoperative day 100 compared with those on postoperative day 7 [71]. MSCs improved liver function and the survival time of rats with LT via downregulation of IL-12 but upregulation of IL-10, TGF- $\alpha_{1}$, TGF- $\beta 1$ and PGE2 [72, 75, 76]. Furthermore, MSCs significantly reduced the acute rejection rate and improved the survival rate of allogeneic LT recipients via downregulating the levels of IL-2, IL-6, IL-17, IL-23, IFN- $\gamma$ and TNF- $\alpha[73,76]$. Chen et al. [77] showed that MSCs significantly eliminated liver allograft rejection and improved the median survival time of LT recipients via upregulation of PD-L1 expression and downregulation of miR-17-5p. On the other hand, injection of MSC-derived conditioned medium inhibited the cell death of LSECs and hepatocytes and promoted liver regeneration, consequently providing additional benefits to the survival rate of rats with reduced-size liver transplantation (RSLT) via downregulating $\mathrm{KC}$ activation, neutrophil infiltration and secretion of inflammatory factors while upregulating the levels of vascular endothelial growth factor (VEGF) and matrix metallopeptidase 9 in the liver grafts [78]. In addition, MSC-derived extracellular vesicles have been proved to exert comparable therapeutic capacities as MSCs themselves in recent years. MSC-derived extracellular vesicles are able to promote the proliferation of endogenous stem cells and tissue regeneration [79]. Furthermore, MSC-derived extracellular vesicles are able to transport noncoding RNAs for the regulation of matrix remodeling, epithelial mesenchymal transitions, resolution of inflammation and immune alleviation [80]. Although there are no studies about the effects of MSCderived extracellular vesicles in improving LT prognosis via their anti-inflammatory effects, MSC-derived extracellular vesicles are worth recognizing as an emerging therapy for treating LT-related immune rejection.

\section{Management of MSC administration in recipients with LT}

Although current studies together showed that MSCs effectively improved LT prognosis after the regulation of immune systems in vivo, the management of MSCs may further improve the therapeutic effects on rejection in liver grafts and recipients. Early treatment with MSC transplantation significantly improved the survival time of rats with acute graft-versus-host disease after LT via upregulation of Treg ratios and Foxp3-positive cells, while late treatment with MSC transplantation from day 8 to day 14 did not attenuate the typical symptoms in rats with acute rejection after LT [81]. This indicates that the time point of MSC transplantation may contribute to the prognosis of LT. To overcome the scarce source of liver grafts, liver donation after cardiac death (DCD) is an alternative approach that may expand the donor pool, while these excised grafts face challenges such as graft dysfunction, early graft loss, and cholangiopathy. Moreover, DCD liver grafts are no longer eligible for 


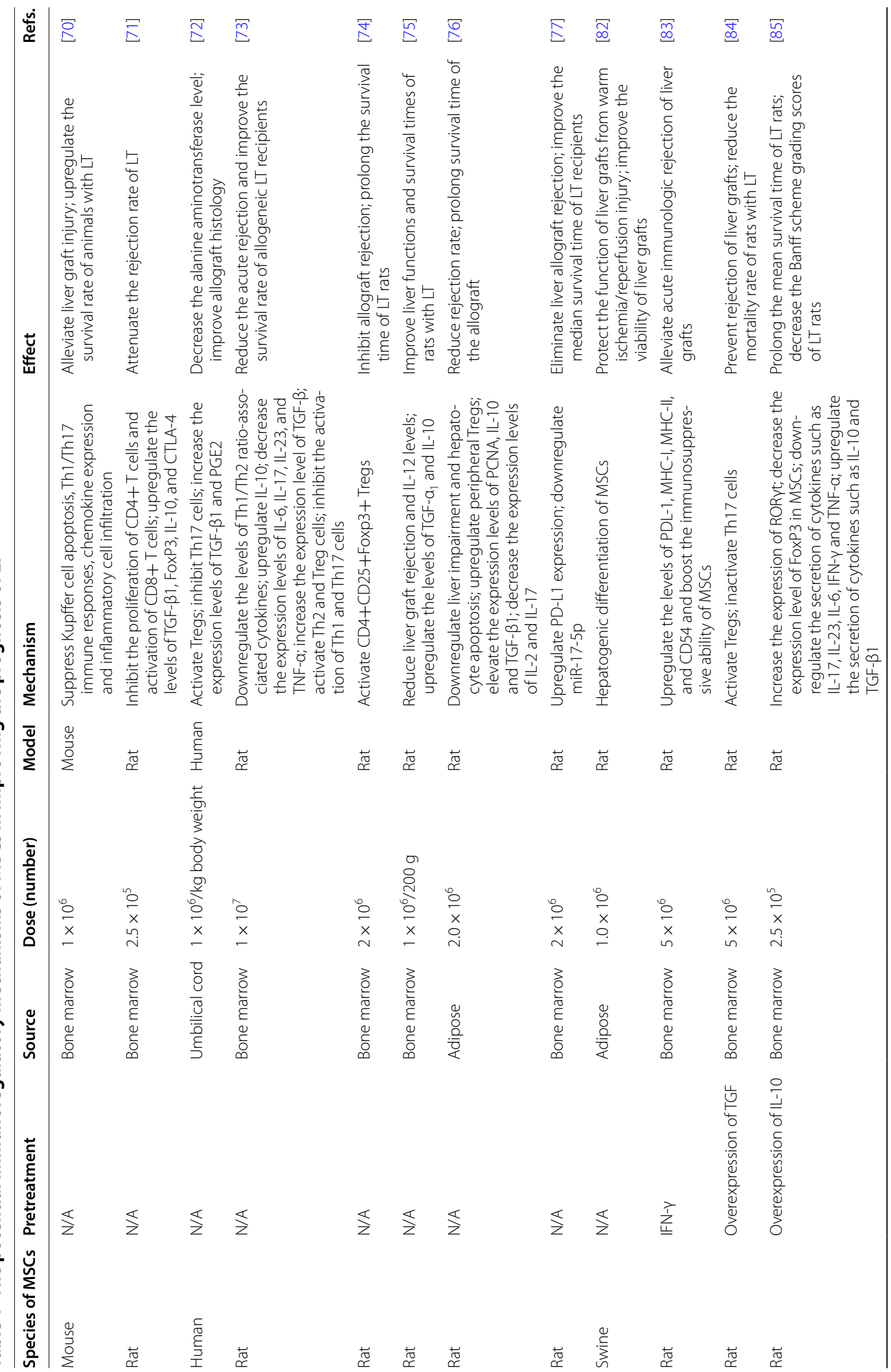




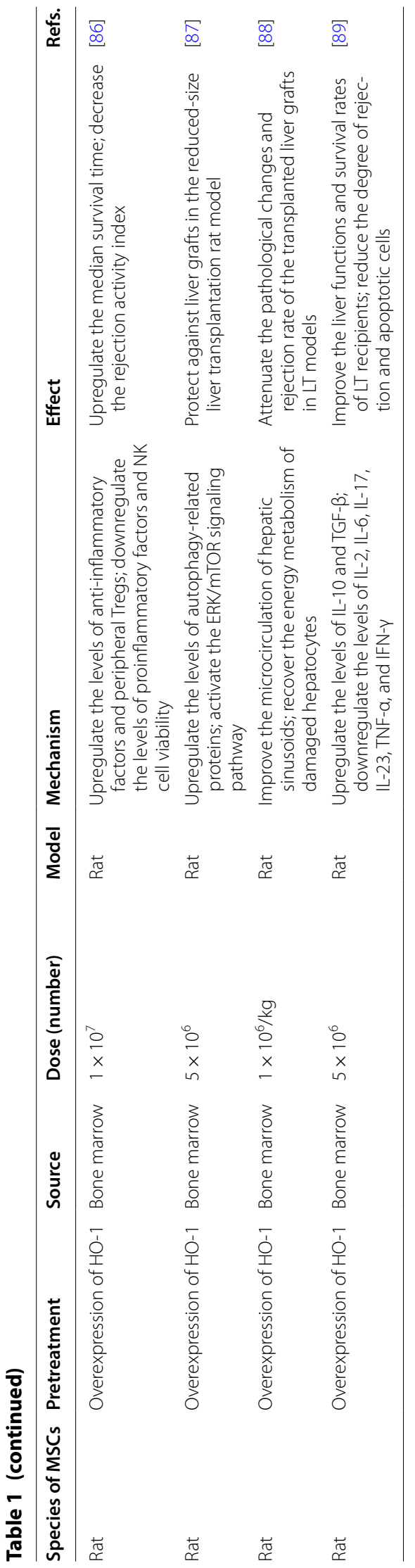




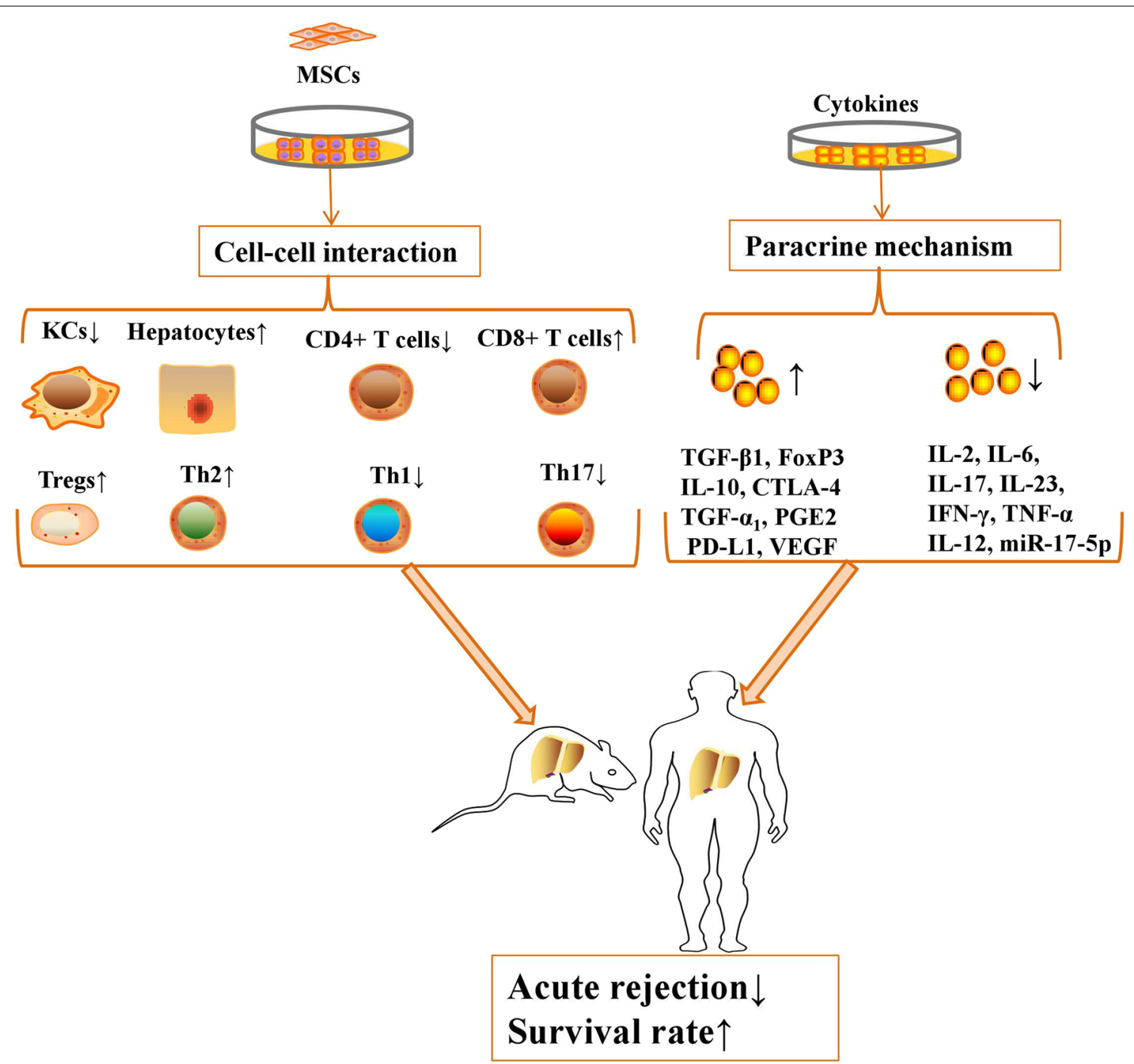

Fig. 2 MSCs exert their immunoregulatory capacities to suppress the immune response by cell-cell interactions and secretion of various cytokines after allogenic LT

transplantation after their warm ischemic time exceeds $30 \mathrm{~min}$. Administration of MSCs protected against warm ischemia-reperfusion injury and protected liver functions of liver grafts from DCD [82].

It is worth highlighting that preconditioning with cytokines or gene modification may enhance the immunoregulatory capacity of MSCs to regulate immune cells in vitro and in vivo. Pretreatment or gene modification of MSCs may serve as an effective method to further improve the immunoregulatory capacity of MSCs in LT. For example, IFN- $\gamma$ pretreated MSCs had upregulated levels of PDL-1, MHC-I, MHC-II, and CD54 and boosted immunosuppressive ability in vivo, consequently improving the homing capacity of MSCs into the liver tissue to alleviate acute immunologic rejection of liver grafts in rats [83]. Transplantation of TGF-overexpressing MSCs also prevented rejection of liver grafts and reduced the mortality rate of rats with LT via activation of Tregs and inactivation of Th17 cells [84]. On the other hand, IL-10-overexpressing MSCs significantly prolonged the mean survival time while decreasing the Banff scheme grading scores of LT rats via upregulation of retinoid acid receptor-related orphan receptor gamma $\mathrm{t}(\mathrm{ROR} \gamma \mathrm{t})$ and downregulation of Foxp3 in MSCs in a time-dependent manner. IL-10 overexpression also downregulated the secretion of cytokines such as IL-6, IL-17, IL-23, IFN- $\gamma$ and TNF- $\alpha$ while upregulating the secretion of cytokines such as IL-10 and TGF- $\beta 1$ in MSCs [85]. Moreover, 
several studies have indicated that heme oxygenase-1 (HO-1) is a key gene in enhancing the immunoregulatory capacity of MSCs in vivo. HO-1-overexpressing MSCs upregulated the median survival time and decreased the rejection activity index via the upregulation of antiinflammatory factors and peripheral Tregs and downregulation of proinflammatory factors and NK cell viability [86]. HO-1-overexpressing MSCs also protected liver grafts against injury in the RSLT rat model via the upregulation of autophagy-related proteins through the ERK/mTOR signaling pathway [87]. Furthermore, overexpression of HO-1 in MSCs improved the microcirculation of hepatic sinusoids and recovered the energy metabolism of damaged hepatocytes via improving the activities of mitochondrial aspartate aminotransferase and ATPase [88]. The immunoregulation of HO-1-overexpressing MSCs is also mediated by the upregulation of IL- 10 and TGF- $\beta$ and the downregulation of IL-2, IL- 6 , IL-17, IL-23, TNF- $\alpha$, and IFN- $\gamma$ to improve allogeneic LT outcomes [89].

To this end, pretreatments with growth factors or gene modification are insufficient to clarify the potential effects of MSC preconditioning on reducing LT-related rejection, due to the scarcity of studies. Thus, it is still obligatory to expand the related studies and explore the potential mechanisms to improve the therapeutic effects of MSCs in the attenuation of graft-versus-host diseases in liver tissue.

\section{Conclusion}

Several types of APCs in the liver and blood circulation recognize external pathogens and subsequently initiate defensive immune mechanisms to attenuate liver inflammation and liver injury. MSCs have been shown to regulate the immune system to attenuate liver inflammation and participate in promoting internal environment homeostasis for the generation of tolerogenic microenvironments in vivo. Striking evidence suggests that MSCs participate in the immunoregulation of liver grafts and recipients to improve the prognosis of LT in preclinical models via cell-cell interactions and anti-inflammatory cytokines. However, pretreatment with anti-inflammatory factors or gene modification of MSCs can further improve the therapeutic effects of MSCs in alleviating the acute graft-versus-host rate. In addition, most gene modifications are overexpression of key genes to enhance the immunoregulatory capacity of MSCs. It is worth considering that knockdown of specific genes in MSCs may also contribute to the improvement of LT prognosis. However, gene modification of MSCs may induce tumorigenesis in recipients after long-term treatment. In the near future, MSC transplantation will further permits a reduction in drug burden and pharmacotherapy inhibitor-associated side effects in LT recipients. However, verification in large-scale and prospective clinical trials has the potential to lead to implementation of MSC protocols in clinical practice for improving LT outcomes.

\section{Abbreviations \\ HCC: hepatocellular carcinoma; LT: liver transplantation; MSC: mesenchymal stem cell; HLA: human leukocyte antigen; MHC: major histocompatibility complex; DCs: dendritic cells; NK cells: natural killer cells; NKT: natural killer T; TNF: tumor necrosis factor; IL: interleukin; TLRs: toll-like receptors; PAMPs: pathogen-associated molecular patterns; DAMPs: damage-associated molecular patterns; APCs: antigen-presenting cells; IFN-Y: interferon-gamma; TCR: T cell receptor; Th1:T helper 1; ILCs: innate lymphocyte cells; ILC1s: group 1 ILCs; GM-CSF: granulocyte-macrophage colony-stimulating factor; BCR: B cel antigen receptor; Tregs: regulatory T cells; Bregs: regulatory B cells; LSECs: liver sinusoidal endothelial cells; KCs: Kupffer cells; TGF: transforming growth factor; iNOS: inducible nitric oxide synthase; NO: nitric oxide; PGE2: prostaglandin E2; RSLT: reduced-size liver transplantation; VEGF: vascular endothelial growth factor; DCD: donation after cardiac death; HO-1: heme oxygenase-1; RORyt: retinoid acid receptor-related orphan receptor gamma t.}

\section{Acknowledgements}

The authors would like to thank the laboratory members for their contributions and the funding support from the sources indicated.

\section{Authors' contributions}

$\mathrm{LL}$ contributed to the conception of this manuscript. $\mathrm{LL}$ and $\mathrm{CH}$ were responsible for the literature review. $\mathrm{CH}$ and $\mathrm{LL}$ drafted and revised the manuscript. Both authors read and approved the final manuscript.

\section{Funding}

This work was supported by the National Natural Science Foundation of China (No. 81700553), the China Postdoctoral Science Foundation (No. 2017M183789), and the Science Fund for Creative Research Groups of the National Natural Science Foundation of China (No. 81121002).

Availability of supporting data

Not applicable.

Ethics approval and consent to participate

Not applicable.

Consent for publication

All authors consent to publication of the present manuscript.

Competing interests

The authors declare that they have no competing interests.

\section{Author details}

${ }^{1}$ Collaborative Innovation Center for Diagnosis and Treatment of Infectious Diseases, State Key Laboratory for Diagnosis and Treatment of Infectious Diseases, First Affiliated Hospital, School of Medicine, Zhejiang University, Hangzhou, Zhejiang, People's Republic of China. ${ }^{2}$ National Clinical Research Center for Infectious Diseases, The First Affiliated Hospital, School of Medicine, Zhejiang University, Hangzhou, Zhejiang, People's Republic of China.

Received: 28 August 2019 Accepted: 4 December 2019

Published online: 10 December 2019

\section{References \\ 1. Meirelles Junior RF, Salvalaggio P, Rezende MB, et al. Liver transplantation: history, outcomes and perspectives. Einstein. 2015;13(1):149-52.}


2. Fisher LR, Henley KS, Lucey MR. Acute cellular rejection after liver transplantation: variability, morbidity, and mortality. Liver Transpl Surg. 1995;1 (1):10-5

3. Calne RY, Rolles K, White DJ, et al. Cyclosporin A initially as the only immunosuppressant in 34 recipients of cadaveric organs: 32 kidneys, 2 pancreases, and 2 livers. Lancet. 1979;2(8151):1033-6.

4. Ascha MS, Ascha ML, Hanouneh IA. Management of immunosuppressant agents following liver transplantation: less is more. World J Hepatol. 2016:8(3):148-61.

5. Dominici M, Le Blanc K, Mueller I, et al. Minimal criteria for defining multipotent mesenchymal stromal cells. The International Society for Cellular Therapy position statement. Cytotherapy. 2006;8(4):315-7.

6. Crisan M, Yap S, Casteilla L, et al. A perivascular origin for mesenchymal stem cells in multiple human organs. Cell Stem Cell. 2008;3(3):301-13.

7. Krampera M, Glennie S, Dyson J, et al. Bone marrow mesenchymal stem cells inhibit the response of naive and memory antigen-specific T cells to their cognate peptide. Blood. 2003;101(9):3722-9.

8. Hu C, Li L. Improvement of mesenchymal stromal cells and their derivatives for treating acute liver failure. J Mol Med. 2019;97(8):1065-84.

9. Le Blanc K, Ringden O. Immunomodulation by mesenchymal stem cells and clinical experience. J Intern Med. 2007;262(5):509-25.

10. Nagy LE. The role of innate immunity in alcoholic liver disease. Alcohol Res. 2015;37(2):237-50.

11. Perdiguero EG, Geissmann F. The development and maintenance of resident macrophages. Nat Immunol. 2016;17(1):2-8.

12. Lavin Y, Mortha A, Rahman A, Merad M. Regulation of macrophage development and function in peripheral tissues. Nat Rev Immunol. 2015;15(12):731-44

13. Ju C, Mandrekar P. Macrophages and alcohol-related liver inflammation. Alcohol Res. 2015;37(2):251-62.

14. Saas $P$, Varin A, Perruche $S$, Ceroi A. Recent insights into the implications of metabolism in plasmacytoid dendritic cell innate functions: potential ways to control these functions. F1000Research. 2017:6:456.

15. Yu H, Tian Y, Wang Y, Mineishi S, Zhang Y. Dendritic cell regulation of graft-vs.-host disease: immunostimulation and tolerance. Front Immunol. 2019;10:93.

16. van den Oord JJ, De Vos R, Facchetti F, et al. Distribution of non-lymphoid, inflammatory cells in chronic HBV infection. J Pathol. 1990;160(3):223-30.

17. Kushwah R, Wu J, Oliver JR, et al. Uptake of apoptotic DC converts immature DC into tolerogenic DC that induce differentiation of Foxp3+ Treg. Eur J Immunol. 2010;40(4):1022-35.

18. Reinders ME, Hoogduijn MJ. NK cells and MSCs: possible implications for MSC therapy in renal transplantation. J Stem Cell Res Ther. 2014;4(2):1000166.

19. Balato A, Unutmaz D, Gaspari AA. Natural killer T cells: an unconventional T-cell subset with diverse effector and regulatory functions. J Invest Dermatol. 2009;129(7):1628-42.

20. Crosby $C M$, Kronenberg M. Tissue-specific functions of invariant natural killer T cells. Nat Rev Immunol. 2018;18(9):559-74.

21. Chapman NM, Shrestha S, Chi H. Metabolism in immune cell differentiation and function. Adv Exp Med Biol. 2017:1011:1-85.

22. Li N, Hua J. Interactions between mesenchymal stem cells and the immune system. Cell Mol Life Sci. 2017:74(13):2345-60.

23. Kaech SM, Cui W. Transcriptional control of effector and memory CD8+ T cell differentiation. Nat Rev Immunol. 2012;12(11):749-61.

24. Albano E. Role of adaptive immunity in alcoholic liver disease. Int J Hepatol. 2012;2012:893026

25. Parra D, Takizawa F, Sunyer JO. Evolution of B cell immunity. Annu Rev Anim Biosci. 2013;1:65-97.

26. Yang $M$, Sun $L$, Wang $S$, et al. Novel function of B cell-activating factor in the induction of IL-10-producing regulatory B cells. J Immunol. 2010;184(7):3321-5.

27. Thomson AW, Knolle PA. Antigen-presenting cell function in the tolerogenic liver environment. Nat Rev Immunol. 2010;10(11):753-66.

28. Invernizzi P. Liver auto-immunology: the paradox of autoimmunity in a tolerogenic organ. J Autoimmun. 2013:46:1-6.

29. Heerwagen C, Schuster M, Bornscheurer A, et al. Rapid exchange of large numbers of donor- and host leukocytes after human liver transplantation. Transpl Int. 2001;14(4):240-7.

30. Sayegh $M H$, Turka $L A$. The role of T-cell costimulatory activation pathways in transplant rejection. N Engl J Med. 1998;338(25):1813-21.
31. Kim HY, Cho ML, Jhun JY, et al. The imbalance of Thelper $17 /$ regulatory $T$ cells and memory B cells during the early post-transplantation period in peripheral blood of living donor liver transplantation recipients under calcineurin inhibitor-based immunosuppression. Immunology. 2013;138(2):124-33.

32. Garcia de la Garza R, Sarobe P, Merino J, et al. Immune monitoring of immunosuppression withdrawal of liver transplant recipients. Transpl Immunol. 2015;33(2):110-6.

33. Jamil KM, Hydes TJ, Cheent KS, et al. STAT4-associated natural killer cell tolerance following liver transplantation. Gut. 2017;66(2):352-61.

34. Obara H, Nagasaki $\mathrm{K}$, Hsieh $\mathrm{CL}$, et al. IFN-gamma, produced by NK cells that infiltrate liver allografts early after transplantation, links the innate and adaptive immune responses. Am J Transplant. 2005;5(9):2094-103.

35. Russo L, Gracia-Sancho J, Garcia-Caldero H, et al. Addition of simvastatin to cold storage solution prevents endothelial dysfunction in explanted rat livers. Hepatology. 2012;55(3):921-30.

36. Limmer A, Ohl J, Kurts C, et al. Efficient presentation of exogenous antigen by liver endothelial cells to CD8+ T cells results in antigen-specific T-cell tolerance. Nat Med. 2000;6(12):1348-54.

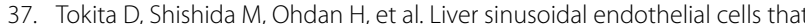
endocytose allogeneic cells suppress T cells with indirect allospecificity. J Immunol. 2006;177(6):3615-24.

38. Kruse N, Neumann K, Schrage A, et al. Priming of CD4+ T cells by liver sinusoidal endothelial cells induces CD25low forkhead box protein 3-regulatory T cells suppressing autoimmune hepatitis. Hepatology. 2009;50(6):1904-13.

39. Karimi MH, Geramizadeh B, Malek-Hosseini SA. Tolerance induction in liver. Int J Organ Transplant Med. 2015;6(2):45-54.

40. Huang HF, Zeng Z, Chen MQ. Roles of Kupffer cells in liver transplantation. Hepatogastroenterology. 2012;59(116):1251-7.

41. Tu Z, Bozorgzadeh A, Pierce RH, et al. TLR-dependent cross talk between human Kupffer cells and NK cells. J Exp Med. 2008;205(1):233-44.

42. Gong J, Cao D, Chen Y, Li J, Zeng Z. Role of programmed death ligand 1 and Kupffer cell in immune regulation after orthotopic liver transplantation in rats. Int Immunopharmacol. 2017:48:8-16.

43. Chen GS, Qi HZ. Effect of Kupffer cells on immune tolerance in liver transplantation. Asian Pac J Trop Med. 2012;5(12):970-2.

44. Breous E, Somanathan S, Vandenberghe LH, Wilson JM. Hepatic regulatory T cells and Kupffer cells are crucial mediators of systemic T cell tolerance to antigens targeting murine liver. Hepatology. 2009;50(2):612-21.

45. Rastellini C, Lu L, Ricordi C, et al. Granulocyte/macrophage colonystimulating factor-stimulated hepatic dendritic cell progenitors prolong pancreatic islet allograft survival. Transplantation. 1995;60(11):1366-70.

46. He W, Chen L, Zheng L, Luo L, Gao L. Prolonged survival effects induced by immature dendritic cells and regulatory T cells in a rat liver transplantation model. Mol Immunol. 2016;79:92-7.

47. Spaggiari GM, Capobianco A, Abdelrazik H, et al. Mesenchymal stem cells inhibit natural killer-cell proliferation, cytotoxicity, and cytokine production: role of indoleamine 2,3-dioxygenase and prostaglandin E2. Blood. 2008;111(3):1327-33.

48. Sotiropoulou PA, Perez SA, Gritzapis AD, Baxevanis CN, Papamichail M Interactions between human mesenchymal stem cells and natural killer cells. Stem Cells. 2006;24(1):74-85.

49. Spaggiari GM, Capobianco A, Becchetti S, Mingari MC, Moretta L. Mesenchymal stem cell-natural killer cell interactions: evidence that activated NK cells are capable of killing MSCs, whereas MSCs can inhibit IL-2-induced NK-cell proliferation. Blood. 2006;107(4):1484-90.

50. Casado JG, Tarazona R, Sanchez-Margallo FM. NK and MSCs crosstalk: the sense of immunomodulation and their sensitivity. Stem Cell Rev. 2013:9(2):184-9.

51. Prigione I, Benvenuto F, Bocca P, et al. Reciprocal interactions between human mesenchymal stem cells and gammadelta T cells or invariant natural killer T cells. Stem Cells. 2009;27(3):693-702.

52. Liu W, Zhang S, Gu S, Sang L, Dai C. Mesenchymal stem cells recruit macrophages to alleviate experimental colitis through TGFbeta1. Cell Physiol Biochem. 2015:35(3):858-65.

53. Francois M, Romieu-Mourez R, Li M, Galipeau J. Human MSC suppression correlates with cytokine induction of indoleamine 2,3-dioxygenase and bystander M2 macrophage differentiation. Mol Ther. 2012;20(1):187-95. 
54. Zhang W, Ge W, Li C, et al. Effects of mesenchymal stem cells on differentiation, maturation, and function of human monocyte-derived dendritic cells. Stem Cells Dev. 2004;13(3):263-71.

55. Spaggiari GM, Moretta L. Interactions between mesenchymal stem cells and dendritic cells. Adv Biochem Eng Biotechnol. 2013;130:199-208.

56. Zhao ZG, Xu W, Sun L, et al. Immunomodulatory function of regulatory dendritic cells induced by mesenchymal stem cells. Immunol Invest. 2012:41(2):183-98.

57. Glennie S, Soeiro I, Dyson PJ, Lam EW, Dazzi F. Bone marrow mesenchymal stem cells induce division arrest anergy of activated T cells. Blood. 2005;105(7):2821-7.

58. Liu Q, Zheng H, Chen X, et al. Human mesenchymal stromal cells enhance the immunomodulatory function of $\mathrm{CD} 8(+) \mathrm{CD} 28(-)$ regulatory T cells. Cell Mol Immunol. 2015;12(6):708-18.

59. Plumas J, Chaperot L, Richard MJ, et al. Mesenchymal stem cells induce apoptosis of activated T cells. Leukemia. 2005;19(9):1597-604.

60. Ren G, Zhang L, Zhao X, et al. Mesenchymal stem cell-mediated immunosuppression occurs via concerted action of chemokines and nitric oxide. Cell Stem Cell. 2008;2(2):141-50.

61. Crop MJ, Baan CC, Korevaar SS, et al. Inflammatory conditions affect gene expression and function of human adipose tissue-derived mesenchymal stem cells. Clin Exp Immunol. 2010;162(3):474-86.

62. Ma S, Xie N, Li W, et al. Immunobiology of mesenchymal stem cells. Cell Death Differ. 2014;21(2):216-25.

63. Prevosto C, Zancolli M, Canevali P, Zocchi MR, Poggi A. Generation of CD4+ or CD8+ regulatory T cells upon mesenchymal stem cell-lymphocyte interaction. Haematologica. 2007;92(7):881-8.

64. Jiang X, Liu C, Hao J, et al. CD4(+)CD25 (+) regulatory T cells are not required for mesenchymal stem cell function in fully $\mathrm{MHC}$-mismatched mouse cardiac transplantation. Cell Tissue Res. 2014;358(2):503-14.

65. Zhang X, Ren X, Li G, et al. Mesenchymal stem cells ameliorate experimental autoimmune uveoretinitis by comprehensive modulation of systemic autoimmunity. Invest Ophthalmol Vis Sci. 2011;52(6):3143-52.

66. Corcione A, Benvenuto F, Ferretti E, et al. Human mesenchymal stem cells modulate B-cell functions. Blood. 2006;107(1):367-72.

67. Peng $Y$, Chen $X$, Liu $Q$, et al. Mesenchymal stromal cells infusions improve refractory chronic graft versus host disease through an increase of CD5+ regulatory B cells producing interleukin 10. Leukemia. 2015;29(3):636-46.

68. Cho DI, Kim MR, Jeong HY, et al. Mesenchymal stem cells reciprocally regulate the M1/M2 balance in mouse bone marrow-derived macrophages. Exp Mol Med. 2014;46:e70.

69. Zhang QZ, Su WR, Shi SH, et al. Human gingiva-derived mesenchymal stem cells elicit polarization of $\mathrm{m} 2$ macrophages and enhance cutaneous wound healing. Stem Cells. 2010;28(10):1856-68.

70. Tian Y, Wang J, Wang W, et al. Mesenchymal stem cells improve mouse non-heart-beating liver graft survival by inhibiting Kupffer cell apoptosis via TLR4-ERK1/2-Fas/FasL-caspase3 pathway regulation. Stem Cell Res Ther. 2016;7(1):157.

71. Niu J, Wang Y, Liu B, Yao Y. Mesenchymal stem cells prolong the survival of orthotopic liver transplants by regulating the expression of TGF-beta1. Turkish J Gastroenterol. 2018;29(5):601-9.

72. Shi M, Liu Z, Wang Y, et al. A pilot study of mesenchymal stem cell therapy for acute liver allograft rejection. Stem Cells Transl Med. 2017;6(12):2053-61.

73. Yang Y, Shen ZY, Wu B, et al. Mesenchymal stem cells improve the outcomes of liver recipients via regulating CD4+ Thelper cytokines in rats. Hepatobiliary Pancreat Dis Int. 2016;15(3):257-65.

74. Wang Y, Zhang A, Ye Z, Xie H, Zheng S. Bone marrow-derived mesenchymal stem cells inhibit acute rejection of rat liver allografts in association with regulatory T-cell expansion. Transplant Proc. 2009;41(10):4352-6.

75. Sun Z, Li T, Wen H, et al. Immunological effect induced by mesenchymal stem cells in a rat liver transplantation model. Exp Ther Med. 2015;10(2):401-6.
76. Gao W, Zhang L, Zhang Y, et al. Adipose-derived mesenchymal stem cells promote liver regeneration and suppress rejection in small-for-size liver allograft. Transpl Immunol. 2017;45:1-7.

77. Chen Q, Zhou R, Zhang Y, et al. Bone marrow mesenchymal stromal cells attenuate liver allograft rejection may via upregulation PD-L1 expression through downregulation of miR-17-5p. Transpl Immunol. 2018;51:21-9.

78. Du Z, Wei C, Cheng K, et al. Mesenchymal stem cell-conditioned medium reduces liver injury and enhances regeneration in reduced-size rat liver transplantation. J Surg Res. 2013;183(2):907-15.

79. Borger V, Bremer M, Ferrer-Tur R, et al. Mesenchymal stem/stromal cellderived extracellular vesicles and their potential as novel immunomodulatory therapeutic agents. Int J Mol Sci. 2017;18(7):1450.

80. Fatima F, Ekstrom K, Nazarenko I, et al. Non-coding RNAs in mesenchymal stem cell-derived extracellular vesicles: deciphering regulatory roles in stem cell potency, inflammatory resolve, and tissue regeneration. Front Genet. 2017:8:161.

81. Xia X, Chen W, Ma T, et al. Mesenchymal stem cells administered after liver transplantation prevent acute graft-versus-host disease in rats. Liver Transpl. 2012;18(6):696-706.

82. Sasajima H, Miyagi S, Kakizaki Y, et al. Cytoprotective effects of mesenchymal stem cells during liver transplantation from donors after cardiac death in rats. Transplant Proc. 2018;50(9):2815-20.

83. Hong ZF, Huang XJ, Yin ZY, Zhao WX, Wang XM. Immunosuppressive function of bone marrow mesenchymal stem cells on acute rejection of liver allografts in rats. Transplant Proc. 2009;41 (1):403-9.

84. Tang J, Yang R, Lv L, et al. Transforming growth factor-beta-expressing mesenchymal stem cells induce local tolerance in a rat liver transplantation model of acute rejection. Stem Cells. 2016;34(11):2681-92.

85. Niu J, Yue W, Song Y, et al. Prevention of acute liver allograft rejection by IL-10-engineered mesenchymal stem cells. Clin Exp Immunol. 2014;176(3):473-84

86. Shen $Z Y, W u B$, Liu T, et al. Immunomodulatory effects of bone marrow mesenchymal stem cells overexpressing heme oxygenase-1: protective effects on acute rejection following reduced-size liver transplantation in a rat model. Cell Immunol. 2017:313:10-24.

87. Wang $R$, Shen $Z$, Yang $L$, et al. Protective effects of heme oxygenase1 -transduced bone marrow-derived mesenchymal stem cells on reducedsize liver transplantation: role of autophagy regulated by the ERK/mTOR signaling pathway. Int J Mol Med. 2017;40(5):1537-48.

88. Yang $L$, Shen ZY, Wang RR, et al. Effects of heme oxygenase-1-modified bone marrow mesenchymal stem cells on microcirculation and energy metabolism following liver transplantation. World I Gastroenterol. 2017;23(19):3449-67.

89. Wu B, Song HL, Yang Y, et al. Improvement of liver transplantation outcome by heme oxygenase-1-transduced bone marrow mesenchymal stem cells in rats. Stem Cells Int. 2016;2016:9235073.

\section{Publisher's Note}

Springer Nature remains neutral with regard to jurisdictional claims in published maps and institutional affiliations.

Ready to submit your research? Choose BMC and benefit from:

- fast, convenient online submission

- thorough peer review by experienced researchers in your field

- rapid publication on acceptance

- support for research data, including large and complex data types

- gold Open Access which fosters wider collaboration and increased citations

- maximum visibility for your research: over 100M website views per year

At BMC, research is always in progress.

Learn more biomedcentral.com/submissions 